\title{
Determining Concentration of Neurotrophic Factors and Neuron Specific Enolase in the Blood of Newborns with Central Nervous System Damages as a New Approach in Clinical Diagnostics
}

DOI: 10.17691/stm2015.7.2.03

Received February 9, 2015

M.V. Vedunova, PhD, Head of the Laboratory for Neuroprotection Methods Development, Nizhny Novgorod Neuroscience Centre, Institute of Living Systems ${ }^{1}$; Senior Researcher, Biochemistry Department, Central Scientific Research Laboratory2;

K.A. Terentieva, PhD Student, Hospital Pediatrics Department²;

N.A. Shchelchkova, PhD, Head of Molecular and Cell Technologies Department, Central Research Laboratory2; Senior Researcher, Laboratory for Neuroprotection Methods Development, Nizhny Novgorod Neuroscience Centre, Institute of Living Systems';

M.A. Kosareva, PhD Student, Hospital Pediatrics Department²;

T.A. Mishchenko, Junior Researcher, Molecular and Cell Technologies Department, Central Research Laboratory; Junior Researcher, Laboratory for Pharmacological Properties of Neurotropic Drugs Research,

Nizhny Novgorod Neuroscience Centre, Institute of Living Systems ${ }^{1}$;

O.V. Khaletskaya, MD, DSc, Professor, Head of Hospital Pediatrics Department;

I.V. Mukhina, DSc, Professor, Head of Central Scientific Research Laboratory2; Head of the Department Normal Physiology named after N.Y. Belenkov2; Professor, Neurodynamics and Neurobiology Department, Biological Faculty ${ }^{1}$; Head of the Laboratory for Pharmacological Properties of Neurotropic Drugs Research, Nizhny Novgorod Neuroscience Centre, Institute of Living Systems ${ }^{1}$

'Lobachevsky State University of Nizhni Novgorod, 23 Prospekt Gagarina, Nizhny Novgorod, 603950,

Russian Federation;

${ }^{2}$ Nizhny Novgorod State Medical Academy, 10/1 Minin and Pozharsky Square, Nizhny Novgorod, 603005,

Russian Federation

The aim of the investigation is to assess the quantity of brain-derived neurotrophic factor (BDNF), glial cell line-derived neurotrophic factor (GDNF) and neuron specific enolase (NSE) in plasma of newborns with perinatal hypoxic damage of CNS.

Materials and Methods. Neurotrophic factors and NSE enzyme concentrations in plasma of newborns (gestation age 31-42 weeks) was studied. The main groups consisted of newborns with the symptoms of perinatal CNS damage (group 1 - with convulsive states, group 2 with the signs of severe perinatal CNS damage), diagnosed according to physical examination, evaluation of the neurological status dynamics and neurosonographic studies. Control group included healthy neonates. Concentration of BDNF, GDNF (R\&D Systems, USA) and NSE enzyme (Vector Best, Russia) was determined by ELISA kit during hospitalization and on day 10-14 after the rehabilitation therapy.

Results. Carried out experiments revealed the significant increase of NSE concentration in plasma of newborns with convulsive states. The higher levels of this enzyme were detected in infants with severe perinatal CNS damage. Moreover, BDNF concentration significantly increases in plasma of patients with the symptoms of severe CNS damage in the period following rehabilitation therapy. These experiments also demonstrate the inverse correlation between BDNF and GDNF levels. It was shown the important prognostic value of BDNF and NSE determination in plasma of newborns with CNS injury.

Conclusion. The most diagnostic value for assessing the severity of brain damage in early neonatal period is associated with measurements of NSE and BDNF concentrations in plasma, which allows to use these markers immediately after birth and before the development of neurological symptoms.

Key words: neuron specific enolase; brain-derived neurotrophic factor; glial cell-line derived neurotrophic factor; hypoxia; perinatal CNS damage.

For contacts: Vedunova Maria Valerievna, e-mail: MVedunova@yandex.ru 
Timely diagnosis and treatment of convulsive states remain an important problem for perinatal neurology and neonatology. The convulsions frequency in neonatal population is $0.5-6 \%$, in prematures $8-12 \%$, in infants with extremely low weight up to $20 \%$ [1]. In neonates, especially in prematures, there is a limited repertoire of neurological symptoms as well as clinical manifestations have similar symptoms in various pathological conditions. In this case, convulsions represent the most evident, clinically delineated and often primary symptom, indicating on dysfunction and damage of the child's central nervous system [2]. Therefore, the data, obtained using modern techniques of central nervous system (CNS) examination, including video-EEG monitoring, neurosonography (NSG), computed tomography (CT) and magnetic resonance tomography (MRT) of the brain are necessary for a deep analysis of clinical parameters in newborns with compulsory assessment. However, it is not always possible to objectively evaluate the CNS condition in newborns as well as the damage severity does not often correspond to the clinical symptoms, especially in premature and the necessity of the gentle treatment makes it impossible to conduct functional diagnostics. Therefore, nowadays it is very important to develop the diagnostic methods based on laboratory tests.

A very promising direction in the early diagnosis of cerebral injuries is immunochemical determination of neurospecific proteins in various biological fluids (blood, liquor). These proteins are tissue-specific for the nervous system and hystogenetically represent the products of neuroepithelial tissue vital activity, i.e. neurons and glial cells. Any pathologic process in the brain leads inevitably to the structural lesions of the nervous tissue and disturbance of the functional integrity of the hematoencephalic barrier, accompanied by the release of the neurospecific proteins into the liquor, and then into the blood. The most significant advantage of the immunochemical neurospecific proteins determination in the biological fluids is high sensitivity, diagnostic accuracy and small amounts $(0.2-0.5 \mu \mathrm{L})$ of the material required for investigation $[3,4]$.

Neuron specific enolase (NSE), brain-derived neurotrophic factor (BDNF) and glial cell line-derived neurotrophic factor (GDNF) are referred to neurospecific proteins, playing a key role in the brain functioning.

BDNF is an important signaling molecule, participating in the regulation of neurogenesis, growth and survival of neurons in CNS. This factor is essential in ischemic and neurodegenerative diseases, and is involved in the protective mechanisms of the nervous tissue in ischemic damage [5].

A representative of transforming growth factor beta family, neurotrophic factor GDNF, is secreted by glial cells (astrocytes, Schwann cells) and promotes preservation, proliferation and differentiation of various cell populations of the central and peripheral nervous system [6, 7]. It is known that the level of GDNF mRNA is dramatically increases and remains elevated for a week after the nervous system damage [8].

NSE is an isoform of enolase enzyme required for glycolysis. Nowadays, NSE is considered as one of the most specific markers of neuron damage, and serves as an indicator of the CNS differentiation degree (NSE is detected at the relatively late stages of neuronal differentiation when synaptogenesis begins, i.e. after the twenty second week of gestation). This is a specific serous marker of neuroendocrine tumors and nervous tissue destruction [9]. NSE level is increased in ischemic lesion and brain trauma, epilepsy, subarachnoid hemorrhage, and is unfavorable prognostic factor of neurological deficiency [10].

The aim of the investigation is to assess the quantity of brain-derived neurotrophic factor, glial cell line-derived neurotrophic factor and neuron specific enolase in plasma of newborns with perinatal hypoxic damage of central nervous system.

Materials and Methods. 17 newborns with gestation age from 31 to 42 weeks were examined. The main group included 10 infants ( 7 boys and 3 girls) with a minimal body weight at birth of $1290 \mathrm{~g}$, treated in the Children's Municipal Clinical Hospital No.1 in Nizhny Novgorod. All infants had the symptoms of severe CNS injury, diagnosed according to physical examination, the assessment of neurological status dynamics and neurosonographic data.

Infants were divided into 2 groups during the observation: group 1 included newborns with neonatal convulsions caused by hypoxia $(n=5)$, group 2 consists of the patients with severe post-hypoxic nervous system damage including atrophic processes formation in the brain without convulsive manifestations $(n=5)$.

Blood samples were collected not later than $72 \mathrm{~h}$ after occurrence of convulsion and 10-14 days after treatment to define the concentration of BDNF, GDNF and NSE enzyme. The obtained plasma was frozen in the $0.5 \mathrm{ml}$ volume and stored at $-20^{\circ} \mathrm{C}$ within 2 months period. Concentration of BDNF, GDNF (R\&D Systems, USA) and NSE enzyme (Vector Best, Russia) was detected by ELISA.

Neuro-psychic status of the newborns was evaluated according to the NACS scale, including the determination of 5 factors: ability to adaptation, passive tone, active tone, primary reflexes, and general condition. The test consists of 20 criteria, each of which is given a score from 0 to 2 . Somatically healthy newborn usually is characterized by the score of 35-40.

According to the history-taking, all children were born from mothers with a complicated course of pregnancy and delivery. The following risk factors were most common: a threat of miscarriage at early terms of gestation, signs of gestosis and insufficient uteroplacental blood circulation, prolonged unhydrous period, labor stimulation. At birth, the condition of all infants was assessed as severe 
or moderately severe. Apgar test at one minute after birth gave the following results: $1-3$ scores in $10 \%$ of newborns, 4-7 scores in 70\%, 7-9 in 20\%. All infants, born in a severe state, were provided with a complex of primary resuscitation measures in the maternity hospital.

Control group comprised of 7 full-term infants without CNS damage. Blood samples from this newborns were taken in the hospital during the required tests according to the indications.

The study complies with the Declaration of Helsinki (the Declaration was passed in Helsinki, Finland, June, 1964, and revised in October, 2000, Edinburg, Scotland) and was performed following approval by the Ethic Committee of Nizhny Novgorod State Medical Academy. Written informed consent was obtained from the patients' parents.

Significance of differences between the experimental groups was defined using a software package ANOVA. Differences were considered significant at $p<0.05$.

Results. Primary assessment neurobehavioral status of infants showed sufficiently low NACS scale (mean 23.2 scores). However, during the treatment and adaptation the score increased to 30.125 .

In group 1 the newborns had focal clonic or generalized tonic convulsions in the first two days of life. Convulsive recurrence was shown in $60 \%$ of infants. For fast termination of convulsive states Diazepam was parenterally injected at dose $0.25-$ $0.50 \mathrm{mg} / \mathrm{kg}$. Phenobarbital was administered per os in repeated cases of convulsions at an initial dose of $10 \mathrm{mg} / \mathrm{kg}$ per day in two divided doses and at a maintenance dose of $5 \mathrm{mg} / \mathrm{kg}$ per day (also double administration). Conducted therapy completely negated the convulsive manifestations in all newborns. In addition, patients were given the necessary symptomatic therapy and treatment aimed to improve metabolic processes in the brain. According to the NSG data posthypoxic changes in all neonates from group 1 were diagnosed. EEG recording did not register any epileptiform activity.

All children from group 2 were born in a heavy state, requiring resuscitation measures and later artificial lung ventilation. NSG and CT revealed the signs of atrophic process development in the brain: periventricular leukomalacia, enlargement of the ventricle size and subarachnoid space. Severe neurological deficiency formed in $40 \%$ of infants over the surveillance period. A moderate delay in neurobehavioral development was noted in other newborns of this group.

Studying the concentration of neurotrophic factors BDNF, GDNF and NSE enzyme in plasma of infants with neonatal convulsions 10-14 days after the start of the treatment the following results were obtained. Statistically significant $(p<0.01)$ increase NSE concentration was noted: this parameter was
2 times higher in comparison with the control group (23.75 \pm 5.49 and $10.12 \pm 1.27 \mathrm{ng} / \mathrm{ml}$ respectively) (See the Figure). The tendency towards the elevation of enzyme concentration in plasmatic fraction was observed in group 2.

Evaluation of neurological status by the NACS scale revealed the high scales of NSE $(27-37 \mathrm{ng} / \mathrm{ml})$ corresponded to less than 20 scores. NSE concentration was significantly decreased $(11-20 \mathrm{ng} / \mathrm{ml})(p<0.05)$ at score more than 20 . These findings indicate the large number of damaged neurons in severe hypoxia.

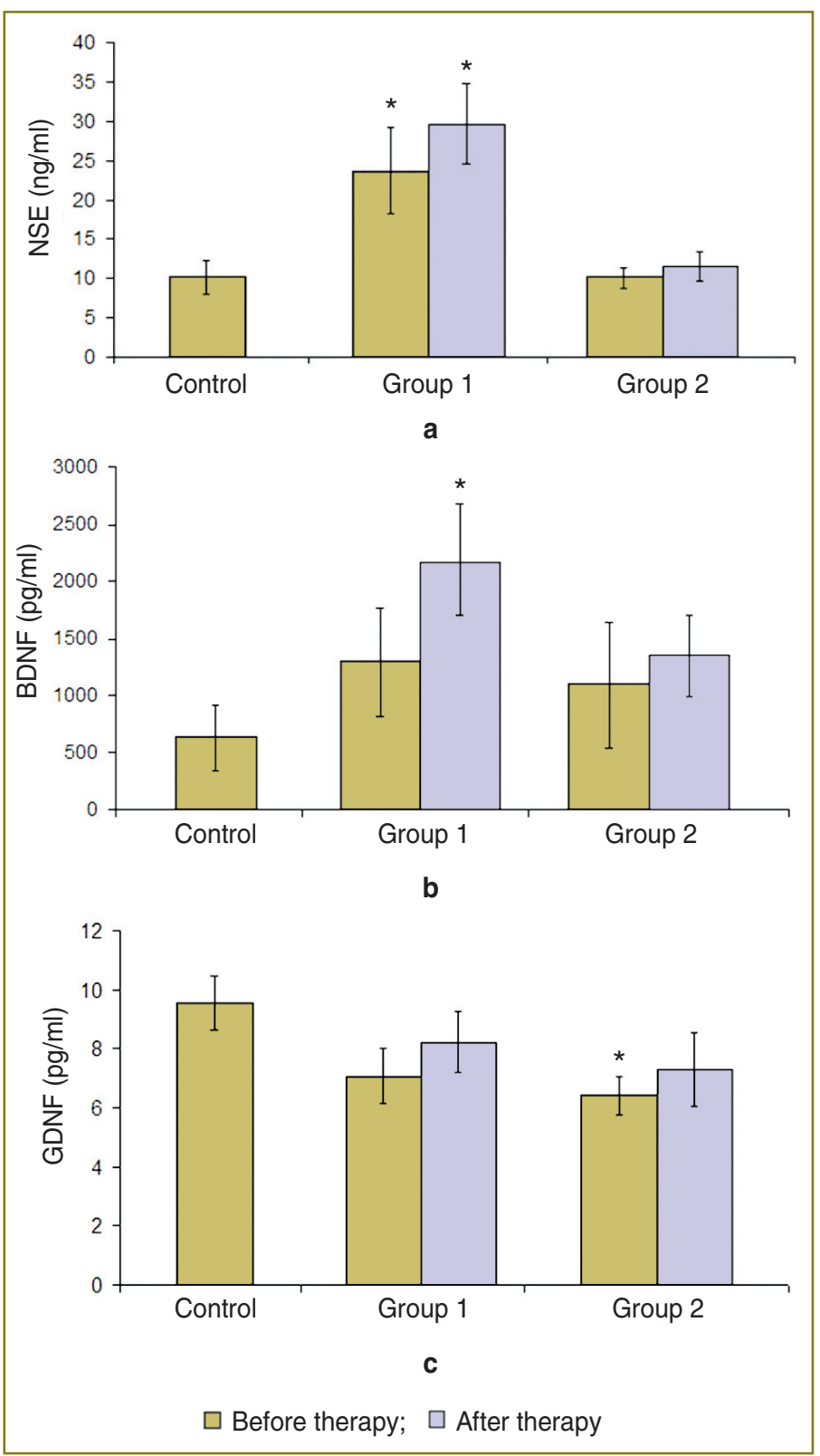

Concentration dynamics of neurotrophic factors BDNF, GDNF and NSE in the plasma of newborns with perinatal hypoxic CNS damage: (a) NSE, * statistical significance with the control group $(p<0.01)$, ANOVA; (b) BDNF (dilution of plasma 1:10), * statistical significance with the control group $(p<0.05)$, ANOVA; (c) GDNF, * statistical significance with the control group $(p<0.01)$, ANOVA 
A direct correlation between NSE concentration and gestation term $(r=0.58)$ in group 1 was noted. Since the value of NSE concentration in plasma is associated with the extent of the brain tissue injury, a large number of damaged brain cells supposed to be observed in infants at later terms of gestation. Different processes take place during the last weeks of pregnancy: differentiation and active development of the brain, growth of neuron connections, brain weight increase as well as the formation of the brain convolutions. However, differentiated neurons with the formed contacts are known to be sensitive to stress-factors [11]. This case hypoxic states involve more neurons at later terms of gestation. And it should be noticed the brain of neonates with the later term of development possesses shows a huge reparation resource and ability to adaptation.

The mean level of NSE in the main groups did not significantly change (NSE concentration in plasma after therapy in group 1 was $29.75 \pm 5.15 \mathrm{ng} / \mathrm{ml}$, in group $2-$ $11.56 \pm 1.83$ ) after a course of treatment. Obtained data suggest prolonged and deep damaging effect of neonatal convulsions and hypoxia on the nervous system of the newborn. Besides, increase of NSE 10-14 days after the beginning of the therapy assumes that hypoxia and convulsive states trigger irreversible processes in nervous system cells, provoking neurons death in the remote period.

Investigation of BDNF concentration in plasma showed a marked tendency towards the increase of this parameter $(p<0.05)$ (in group 1 it amounted to $36.87 \%$, in group $2-22.01 \%$ ) in both groups $10-14$ days after the beginning of the therapy.

This effect in combination with anticonvulsants may be considered as an adaptive reaction of the body to the stress. Since the main pharmacological target of the anticonvulsive medicines is to increase GABA system activity, BDNF elevation assumes the compensatory reaction to the introduction of substances that regulate the activity of GABA-receptors. According to the previously data $[11,12], B D N F$ is able to influence the GABA-receptors endocytosis via tropomyosin-related kinase $B$ receptor, controlling the number this type of the receptor on the plasma membrane of cells.

The obtained interconnection between BDNF concentration in plasma and neurological status of the patients after the course of treatment $(r=-0.81)$ is especially interested. Thus, it was shown the prognostic

\section{Correlation coefficient between the concentrations} of neurotrophic factors BDNF and GDNF in the plasma of newborns

\begin{tabular}{|lcc|}
\hline \multicolumn{1}{|c}{ Groups } & Before therapy & After therapy \\
\hline Control (healthy newborns) & -0.65 & - \\
\hline Experimental group 1 & -0.91 & -0.52 \\
\hline Experimental group 2 & -0.90 & -0.07 \\
\hline
\end{tabular}

value of this parameter for clinical diagnostics. At the same time, NSE concentration (the main biochemical parameter of CNS damage) at the beginning of the therapy is not associated with the success of the medical treatment and alteration of the patient neurological status. The obtained dependence indicates the diagnostic value of determining BDNF concentration in infants with severe CNS disorders. Moreover, initial BDNF increase over $4000 \mathrm{pg} / \mathrm{ml}$ (dilution of plasma 1:10) is an unfavorable prognostic symptom. Such elevation may be considered as a decompensatory reaction. In this subgroup of patients the inefficiency of the applied therapy and the development of neurological disturbances were registered.

At the same time, GDNF concentration in plasma in both main groups of the infants before treatment was lower than in the control one that is probably caused to the suppression of the nervous tissue growth after severe hypoxia: it was $9.55 \pm 0.66 \mathrm{pg} / \mathrm{ml}$ in the control group, $7.09 \pm 0.07 \mathrm{pg} / \mathrm{ml}$ in the group with convulsions, and $6.43 \pm 0.63 \mathrm{pg} / \mathrm{ml}$ in group 2 .

The significant correlation between neurotrophic factors (BDNF, GDNF) concentrations $(r=-0.65)$ in plasma of newborns in the control group were detected (See the Table). In the main groups 1 and 2 stronger correlation of neurotrophic factors is noted $(r=-0.91$ in group 1, $r=-0.90$ in group 2), which became lower after the course of treatment due to the increase of BDNF concentration ( $r=-0.52$ in group 1 , and in group 2 the correlation is lost, $r=-0.07$ ). The obtained data are fully confirmed by the experimental studies, investigating the role of neurotrophic factors BDNF and GDNF in the neuron networks protection of dissociated hippocampal cultures from hypoxic state [13]. It was previously shown that each neurotrophic factors have strong neuroprotective effect, but their combined application reduces the positive individual effect of the neutrophins.

Strengthening of the negative correlation between neurotrophic factor concentrations in developing convulsive states indicates that intracellular metabolic cascades, activated by BDNF and GDNF, are differently directed in stress condition. Decrease in the correlation coefficient after therapy is probably caused by the activation of BDNF synthesis following the anticonvulsants application.

Discussion. Investigations of recent years have proved the key role of the neutrophic factors not only in the prenatal neurogenesis, but in postnatal period as well. Signal pathways, activated by the brain-derived neurotrophic factor and glial cell line-derived neurotrophic factor, are different, but directed to increase neuron survival in stress and reduce inflammatory reactions.

These studies demonstrate that both anticonvulsive and neurometabolic therapies result in the increase of BDNF concentration in plasma. This factor plays an important role in forming adaptive reactions affected by 
severe perinatal hypoxia. The main molecular target of BDNF is tropomyosin-related kinase $B$ receptor (TrkB). Interaction between a mature BDNF molecule and TrkBreceptor triggers intracellular signaling mechanisms, leading to the growth of axons and dendrites, increase of cell survival, neuron plasticity, BDNF transcription. According to the localization of the TrkB-receptor in axonal terminals and dendrite spines, BDNF influences the presynaptic and postsynaptic transmission [14]. Recent investigations $[11,15]$ showed a positive feed-back between GABAergic system and BDNF synthesis level. $\mathrm{Ca}^{2+}$ influx into the cell after GABA-receptor activation stimulates BDNF release and its binding to the TrkBreceptor with the following triggering of phosphoinositol-3 and proteinkinase-C signaling pathways, resulting in the increase of GABAA-receptor expression. Once signal cascades are triggered, BDNF decreases endocytosis of GABAA-receptors, increasing their quantity on the cell surface, which leads to intensifying the sensitivity of the developing neurons to GABA [11, 15].

Thus, elevation of BDNF concentration may be considered, on the one hand, as a compensatory reaction of the organism to severe chronic hypoxia, and on the other, may serve one of the links of metabolic respond to the medicines, influencing the synaptic transmission. Of special interest are data on correlation between BDND and GDNF concentration. According to the established concept $[11,15]$, both neurotrophic factors play a key role in the differentiation of neurons and development of adequate synaptic contacts. This approach is realized in differentiation methods and proliferation of neuronal progenitors, including induced pluripotent stem cells [16]. However, experimental studies [17] showed, that the effects of BDNF and GDNF have differently directed character, and combined application of neurotrophic factors reduces their efficacy.

Conclusion. Neuron specific enolase is the most expressive marker of nervous tissue damage. A high level of NSE enzyme is noted in infants, suffered neonatal convulsions, testifying to the marked damaging effect of convulsions on the nervous system of neonates. NSE concentration correlates with the neurological status of the patients and keeps rising in $62.5 \%$ of newborns even after a course of treatment, which supposed to be the evidence of the continuing neuron destruction. BDNF concentration in plasma of newborns with severe CNS disturbance also significantly increases after therapy. The detected correlation between the initial level of this neutrophin and patient condition (assessment of neurological status) after treatment proves the diagnostic value of BDNF determination. Significant changes in the GDNF concentration in the plasma of newborns with severe CNS disorders have not been established.

Thus, the most diagnostic value for assessing the severity of brain damage at an early stage of the neonatal period is associated with measurements of NSE and BDNF concentrations in plasma, which allows to use these markers immediately after birth and before the development of neurological symptoms.

Study Funding. The study was supported by the State represented by the Russian Ministry of Education and Science in the frames of the project of the Federal purpose-oriented program "Unique scientific device for investigation of the information processes in the brain using optogenetic techniques"; unique identifier of the project RFMEFI 59114X0004, agreement on financial granting of Jan. 01, 2014 between the Ministry of Education and Science of the Russian Federation and Lobachevsky State University of Nizhni Novgorod with application of the equipment of the unique scientific device.

Conflict of Interests. The authors have no conflict of interests to disclose.

\section{References}

1. Tharp B.R. Neonatal seizures and syndromes. Epilepsia 2002; 43(Suppl 3): 2-10, http://dx.doi.org/10.1046/j.15281157.43.s.3.11.x.

2. Volpe Y. Neurology of newborn. N.Y.; 2002.

3. Volodin N.N., Degtyarev D.N., Khachatryan A.V., Khokhlov A.P., Navasardyants D.G. Change of neuron specific protein content - neuron specific enolase, leucine aminopeptidase, cytokine tumor necrosis factor $\alpha$ in infants with perinatal CNS damage. Pediatriya 1998; 5: 15-20.

4. Turina O.I., Ryabukhin I.A., Rogatkin S.O., Shepeleva I.I., Degtyarev D.N., Anin A.N., Chekhonin V.P., Volodin H.H. Enzyme immunodetection of the level of gliofibrillar acid protein and antibodies against it in the assessment of perinatal CNS damages in preterm infants. Pediatriya 1995; 3: 15-19.

5. Fantacci C., Capozzi D., Ferrara P., Chiaretti A. Neuroprotective role of nerve growth factor in hypoxic-ischemic brain injury. Brain Sci 2013; 3(3): 1013-1022, http://dx.doi. org/10.3390/brainsci3031013.

6. Chen Y. Effects of glial cell line-derived neurotrophic factor (GDNF) on stem/progenitor cell proliferation and differentiation. Abstract of dissertation. Lexington, Kentucky; 2005.

7. Airaksinen M.S., Saarma M. The GDNF family: signalling, biological functions and therapeutic value. Nat Rev Neurosci 2002; 3(5): 383-394, http://dx.doi.org/10.1038/nrn812.

8. Ahmed F., Gyorgy A., Kamnaksh A., Ling G., Tong L., Parks S., Agoston D. Time-dependent changes of protein biomarker levels in the cerebrospinal fluid after blast traumatic brain injury. Electrophoresis 2012; 33(24): 3705-3711, http:// dx.doi.org/10.1002/elps.201200299.

9. Singh H.V., Pandey A., Shrivastava A.K., Raizada A., Singh S.K., Singh N. Prognostic value of neuron specific enolase and IL-10 in ischemic stroke and its correlation with degree of neurological deficit. Clin Chim Acta 2013; 419: 136138, http://dx.doi.org/10.1016/j.cca.2013.02.014.

10. Olenev S.N., Olenev A.S., Neronova Yu.I. Evolyutsiya mozga cheloveka [Evolution of the human brain]. Saint Petersburg: Nestor; 2000.

11. Sakharnova T.A., Vedunova M.V., Mukhina I.V. Brain-derived neurotrophic factor (BDNF) and its role in the functioning of the central nervous system. Neurochemical 
Journal 2012; 6(4): 251-259, http://dx.doi.org/10.1134/ s1819712412030129.

12. Vedunova M.V., Sakharnova T.A., Mitroshina E.V., Mukhina I.V. The role of the tropomyosin-related kinase $B$ (TrkB) receptor in realization of neuroprotective and antihypoxic properties of brain-derived neurotrophic factor (BDNF) during normobaric hypoxia in vitro. Biomeditsinskaya radioelektronika 2014; 4: 13-14.

13. Lu B. Pro-region of neurotrophins: role in synaptic modulation. Neuron 2003; 39(5): 735-738, http://dx.doi. org/10.1016/s0896-6273(03)00538-5.

14. Song D.K., Choe B., Bae J.H., Park W.K., Han I.S., Ho W.K., Earm Y.E. Brain-derived neurotrophic factor rapidly potentiates synaptic transmission through NMDA, but suppresses it through non-NMDA receptors in rat hippocampal neuron. Brain Res 1998; 799(1): 176-179, http://dx.doi. org/10.1016/s0006-8993(98)00474-0.

15. Tanaka T., Saito H., Matsuki N. Inhibition of $G A B A_{A}$ synaptic responses by brain-derived neurotrophic factor (BDNF) in rat hippocampus. J Neurosci 1997; 17(9): 29592966.

16. Porcher C., Hatchett C., Longbottom R.E., McAinch K., Sihra T.S., Moss S.J., Thomson A.M., Jovanovic J.N. Positive feedback regulation between gamma-aminobutyric acid type $A\left(G A B A_{A}\right)$ receptor signaling and brain-derived neurotrophic factor (BDNF) release in developing neurons. J Biol Chem 2011; 286(24): 21667-21677, http://dx.doi.org/10.1074/jbc. $\mathrm{m} 110.201582$.

17. Lebedeva O.S., Lagar'kova M.A., Kiselev S.L., Mukhina I.V., Vedunova M.V., Usova O.V., Stavrovskaya A.V., Yamshchikova N.G., Fedotova E.Y., Grivennikov I.A., Khaspekov L.G., Illarioshkin S.N. The morphofunctional properties of induced pluripotent stem cells derived from human skin fibroblasts and differentiated to dopaminergic neurons. Neurochemical Journal 2013; 7(3): 207214, http://dx.doi.org/10.1134/s1819712413030082. 